\title{
INTERACTIVE TV USER EXPERIENCE IN BEHAVIORAL SITUATIONS
}

\author{
Seungyeon Lee and Myunghwan Yun \\ Department of Industrial Engineering, Seoul National University \\ 1 Gwanak ro, Gwanak-gu, Seoul 08826, South Korea
}

\begin{abstract}
Television (TV) is one of the most familiar products in our lives. As TVs evolve into interactive products, the user experience (UX) surrounding TV is also changing. Interactive TV attributes provide new capabilities; however, it is not clear how this affects the TV viewer's experience. The perceived quality of interactive products has two sub-dimensions: ergonomic attributes and hedonic attributes. Users perceive the quality of interactive products differently according to behavioral situations, and have overall experiences through a combination of those sub-dimensions. We hypothesize that a match between an individual's behavior and a particular product's attributes (i.e., ergonomic, hedonic) determine the perceived value of interactive products. Subsequently, we conducted a study with eighty TV viewers, in which half were asked to perform goal-directed tasks with interactive TV and the other half were asked to perform experiential tasks. The results obtained show that interactive TV viewers find interactive TV more appealing when they perform goal-directed behaviors. Regardless of the purpose of use, hedonic attributes were higher than ergonomic attributes on the perceived value of interactive TV. This implies the fundamental purpose of the user-TV interaction is to experience pleasure and achieving the goal is also the pleasure-seeking process as part of the fun. This also implies that hedonic aspect is the important factor than the ergonomic aspect on the TV-viewing experience regardless of the situation. The results demonstrate the potential value of hedonic quality as a measurement factor of interactive TV user experience evaluation and provide implications that interactive TV UX should be designed to enhance the enjoyable experience.
\end{abstract}

\section{KEYWORDS}

Interactive TV, User Experience (UX), Goal-Directed, Experiential, Hedonic Quality, Ergonomic Quality

\section{INTRODUCTION}

Television (TV) has been loved for a long time as an entertainment medium for relaxation and fun. As TV has become connected to the Internet, and the TV platform has developed to accommodate a variety of applications, it is increasingly becoming an active and interactive medium and the experience of TV viewers is also changing. The key features of interactive TV are, however, focused on new business opportunities (Bellman et al. 2017; Lekakos et al. 2001) or its functional aspects (Morris and Smith-Chaigneau 2012; O'Sullivan et al. 2004), rather than investigating TV viewer's entertainment needs (Chorianopoulos and Spinellis 2004; Livaditi et al. 2003). This diverse function and active usage strengthened by industrial developments obviously differentiates interactive TV from the previous non-interactive TVs (hereinafter "legacy TV"). However, not many studies convince that interactive TV enhances the TV viewer's experience. Instead, there are contrary studies that TV viewers rarely use the interactive TV's new features because they are unnecessary or difficult to use (Darnell 2007; Lee and Lee 1995).

The continuous diffusion of interactive products is possible when people use them casually and frequently. Ardito et al. (2008) stated that experiences are influenced "by the user's psychological state and the context" as well as the characteristics of the interactive system. Chorianopoulos and Spinellis (2004) argued that the evaluation of the traditional user interface might not be adequate for assessing TV applications. Therefore, interactive TV research should also examine the user's experience in terms of "everyday use."

Many studies in human-computer interaction (HCI) have focused on the usability aspects of interactive products. However, studies on everyday products like TV, which are more focused on non-utilitarian tasks, have begun to emphasize the importance of hedonic judgements as important as usability for the overall user 
experience (Hassenzahl et al. 2000; Hassenzahl and Ullrich 2007; Mano and Oliver 1993). According to those studies, people perceive interactive products in terms of both their ergonomic qualities (EQ) and hedonic qualities (HQ). Hedonic aspects and pragmatic aspects are sub-properties that describe a user's experience, and the user's overall experience is a combination of these sub-experiences. The pragmatic aspects mainly involve the product's utility and usability, including it being simple and predictable. The hedonic aspects include aesthetics, self-assessment, relatedness, novelty, and so on (Jordan 2000). According to Hassenzahl's user experience (UX) research model (2000), the objective quality of the product was judged through the user's perceived hedonic quality and the ergonomic quality and the process of making the overall judgment.

There are also other claims that non-utilitarian factors, such as fun, enjoyment, or aesthetics, are the main factors that affect the user experience (Hartmann et al. 2007; Norman 2004; Tractinsky et al. 2000; Tuch et al. 2012). Tractinsky et al. (2000) argued that the more beautiful the design, the more useful the result. Norman (2004), who emphasized the importance of emotional factors, argued that non-utilitarian aspects play a mediating role in goal-directed activity rather than usability, because the emotional impact through the aesthetic factor has a greater effect on the user experience. Dillon (2002) insisted that aesthetics work as a "driver," enhancing usability. This argument is more convincing as the range of interactive media is increasingly expanding. Bernhoupt and Pirker (2013) also asserted that aesthetics, emotion and stimulation are important UX factors on the interactive TV domain.

The user experience is affected not only by the product's own attributes but also by the user's behavior. According to Apter's (1989) reversal theory, users are affected more pragmatically when they are unconsciously or consciously exposed to a goal-oriented situation. Conversely, if a behavior itself is the goal, the hedonic aspects become more crucial factors to the users. Many studies on consumer experiences in online environments indicate that there are two types of consumption behavior: goal-directed and experiential (Novak et al. 2003; Sánchez-Franco and Roldán 2005). According to Novak et al. (2003), these two categories of behavior have distinct different attributes in important consumer behavior issues such as involvement, search and decision making. Goal-directed behaviors have the following attributes: extrinsic, directed search, and planned purchases. Meanwhile, experiential behaviors have the following attributes: intrinsic, ongoing search, and impulse buys. Hassenzahl et al. (2002) found that the users' evaluations varied depending on whether they had a goal to achieve or not.

TV has been classified as a passive entertainment medium providing relaxation and enjoyment. Interactivity enables more active user participation. People may want to use interactive TV more extensively. On the contrary, people may prefer to just watch TV or may not like the interactive attributes of TV. The purpose of this study is to identify opportunities to see how interacting with TV in the different situations affects the TV viewer's experiences. To do this, we assumed that the match between an individual's motivational orientation and a particular product attribute (i.e., ergonomic or hedonic) would determine the perceived value of the interactive product, and we applied this to interactive TV viewers.

\subsection{Research Questions and Hypothesis}

The main research question is, when using interactive TV, whether users experience differences between goal-directed behavior and experiential behavior. To answer this research question, two hypotheses were chosen:

H1. The experience aspects users consider will differ between goal-directed situations and experiential situations.

H2. Users will be more appealed in the goal-directed situation than in the experiential situation.

Another research question is what is the main factor affecting the interactive TV-viewing experience? Based on the question, one more hypothesis was set up:

$\mathrm{H} 3$. Hedonic quality will affect the interactive TV-viewing experience more than ergonomic quality. 


\section{METHODS}

\subsection{User Experience Model}

In this study, we employed Hassenzahl and his colleagues' user research model (2000) and adopted it to different situations to investigate how interactive TV viewers' experiences are differentiated in behavioral situations. Hassenzahl et al. (2000) explained that a product has different quality dimensions and suggested a user experience research model consisting of four steps: objective quality, perceived quality, evaluation of the software, and consequences of using the software (or product). They proposed that the process through which the software appeals to users consists of the users' perceptions, evaluation, and the consequences of using the software.

Before starting the main study, we conducted a survey to determine the main behavior of TV viewers. We asked participants about the main purpose of watching TV $(\mathrm{N}=$ ninety-nine: female = fifty-eight, male $=$ forty-one, average age $=$ thirty-six years). We created a questionnaire about watching TV and administered the survey through SurveyMonkey.com (San Mateo, CA, USA), which is an online survey site. This survey was conducted for four days in 2017. It was found that sixty-eight percent of the respondents habitually turn on the TV to pass time, or to find "something fun." They search for a TV program by "channel zapping" or by navigating the menu. The average search time was three to five minutes. In addition, thirty-one percent said that they turn on the TV to watch a particular program. They preferred to reach the target content directly and quickly. The average time to reach the intended content was one to two minutes. The results showed that differences exist in the TV viewers' behavior and interaction time based on whether they have a goal. TV viewers usually find interesting content through browsing or menu navigating, whereas when they have specific target content, they go directly to the destination. (Figure 1).

In this experiment, the interactive TV service called Olleh TV, which is provided by Korea Telecom (KT), was used as the material (Figure 1). Olleh TV is a market-leading Internet protocol TV service in Korea that provide thousands of live channels, video content, and applications. The Olleh TV service interface has a hierarchical menu structure. Using a remote control, users navigate the menu in a vertical direction to first search for a category and then select sub-content belonging to the category in a horizontal direction.

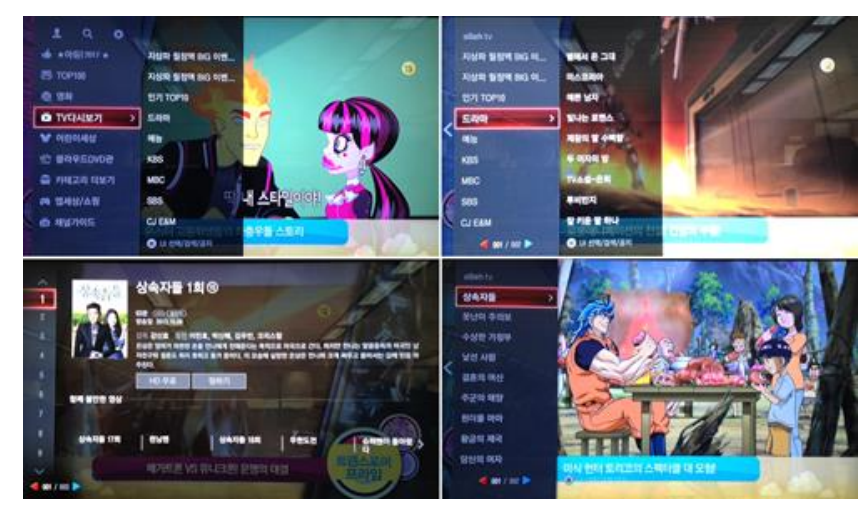

Figure 1. The hierarchy type user interface of Kore Telecom's interactive television service ("Olleh TV"). Clockwise from top left: 1 depth menu, 2 depth menu, 3 depth menu, and content page. Up and down keys on the remote control are used to select categories in the same depth, and left and right keys are used to move between depths

Eighty participants took part in this study (male $=$ forty-four, female $=$ thirty-six, mean age $=$ twenty-nine years). All participants were new employees of KT. We recruited the participants from departments that were not directly related to the interactive TV service to avoid distorting the results of the experiment. All participants were familiar with the use of a remote control and knew how to search for channels and find content. No participation fee was paid to them. To allow participants to watch and manipulate the TV comfortably, a separate room with a comfortable chair was used. The distance between the TV and participants was about two meters. A fifty-inch high definition TV was used in the experiment. 


\subsection{Measurement of the Hedonic Quality, Ergonomic Quality and Appeal}

Regarding the scale for measuring the HQ, EQ, and appeal in terms of the user's experience, we employed the Semantic Differential Questionnaire presented by Hassenzahl (2001). The Semantic Differential Questionnaire is a rating scale to measure the connotative meaning of products and concepts. The hedonic quality (HQ) is composed of seven questions and consists of emotional adjectives such as "interesting - boring," "exciting - dull" and so on. Ergonomic quality (EQ) is composed of eight questions about usability aspects such as "comprehensible - incomprehensible" or "supporting - obstructing." APPEAL is the integrated user feeling consisting of perceived HQ and EQ and has eight questions on dimensions such as "pleasant - unpleasant," or "good - bad." Each item consists of 7 points from -3 to +3 , which means that the larger the negative value, the more the users are positively experienced.

\subsection{Procedure}

All participants signed an agreement to participate before starting the experiment. Eighty participants were divided into two groups. The first group performed three specific goal-directed tasks: (1) searching for a specific program's title through menu navigation, (2) finding specific video-on-demand content, and (3) registering their preferred channels. Each task's time was limited to three minutes in the goal-directed session. The experimental difficulty was adjusted appropriately so that all participants could complete the task in time. We counterbalanced the task order for the goal-directed group. After completing the assignment, the participants completed the semantic differential questionnaire about the perceived quality of the product and SAM questionnaire about their feeling. The second group performed the experiential tasks for ten minutes of freely navigating channels and finding content as they normally do when using a TV. After using the TV, participants also answered the same questionnaires mentioned above.

\section{RESULTS}

A factor analysis was performed to determine whether TV viewers perceived ergonomic quality (EQ) and hedonic quality (HQ) independently in each task. The factor analysis extracted two EQ factors and a main HQ factor from the semantic differential for the goal-directed tasks. All the sub-attributes of HQ were similar to one another and the eigenvalue of the extracted factor was $6.39(42.64 \%)$; the explanatory range was $42.64 \%$ of the total variance. The eigenvalue of factor 1 extracted from EQ was $3.34(22.26 \%)$ and factor 2 was $1.95(13 \%)$; both factors accounted for $35.26 \%$ of the total variance. The combined EQ and HQ factors explained $78 \%$ of the total variance (see Table 1 ).

Table 1. Factorial validity of the goal-directed tasks' ergonomic quality and hedonic quality

\begin{tabular}{llrr}
\hline The user perceived product's attributes & Principal Components with Varimax & \\
& Factor 1 & Factor 2 & Factor 3 \\
\hline G_HQ6 original - ordinary & .969 & -.039 & -.053 \\
G_HQ5 impressive - nondescript & .930 & .163 & .068 \\
G_HQ4 exclusive - standard & .927 & -.029 & .003 \\
G_HQ7 innovative - conservative & .924 & .136 & -.067 \\
G_HQ3 exciting - dull & .919 & .159 & -.031 \\
G_HQ2 costly - cheap & .870 & .030 & .072 \\
G_HQ1 interesting - boring & .852 & .171 & -.101 \\
G_EQ1 comprehensible - incomprehensible & .094 & .840 & .259 \\
G_EQ3 simple - complex & .250 & .834 & -.005 \\
G_EQ7 controllable - uncontrollable & .074 & .794 & .202 \\
G_EQ8 familiar - strange & -.424 & .642 & .025 \\
G_EQ2 supporting - obstructing & .516 & .633 & .149 \\
G_EQ4 predictable - unpredictable & -.068 & .310 & .852 \\
G_EQ6 trustworthy - shady & -.063 & .030 & .852 \\
G_EQ5 clear - confusing & .128 & .544 & .585 \\
Eigenvalue & 6.396 & 3.340 & 1.951 \\
\% variance explained & 42.641 & 22.265 & 13.007 \\
\hline
\end{tabular}


A factor analysis for the experiential tasks extracted a main EQ factor and a main HQ factor from the semantic differential. The eigenvalue of EQ factor was $5.52(36.81 \%)$ and HQ was $5.39(35.96 \%)$ and the combined result explained $73 \%$ of the total variance (see Table 2 ).

Table 2. Factorial validity of the experiential tasks' ergonomic quality and hedonic quality

\begin{tabular}{llr}
\hline The user perceived product's attributes & \multicolumn{2}{l}{ Principal Components with Varimax } \\
& Factor 1 & Factor 2 \\
\hline E_EQ5 clear - confusing & & .850 \\
E_EQ4 predictable - unpredictable & .785 & .072 \\
E_EQ1 comprehensible - incomprehensible & .782 & .154 \\
E_EQ8 familiar - strange & .885 & -.161 \\
E_EQ2 supporting - obstructing & .886 & -.214 \\
E_EQ3 simple - complex & .697 & 0.1 \\
E_EQ7 controllable - uncontrollable & .726 & .078 \\
E_EQ6 trustworthy - shady & .804 & -.175 \\
E_HQ4 exclusive - standard & .133 & -.142 \\
E_HQ7 innovative - conservative & .378 & .789 \\
E_HQ6 original - ordinary & .1 & .809 \\
E_HQ5 impressive - nondescript & -.26 & .877 \\
E_HQ3 exciting - dull & -.252 & .905 \\
E_HQ2 costly - cheap & -.112 & .879 \\
E_HQ1 interesting - boring & -.169 & .885 \\
Eigenvalue & 5.522 & .898 \\
\% variance explained & 36.814 & 5.394 \\
\hline
\end{tabular}

Table 3 shows that each variable had high internal consistency and was reliable to use as a variable in this experiment (Cronbach's $\alpha$ : EQ $=.922 ; \mathrm{HQ}=.944$; Appeal $=.958$ in experiential tasks; Cronbach's $\alpha: \mathrm{EQ}=.840 ; \mathrm{HQ}=.970 ;$ Appeal $=.958$ in goal-directed tasks). According to the results, the HQ, EQ and Appeal were experienced more in the goal-directed tasks (EQ Mean = -1.362; HQ Mean = -.507; Appeal Mean = -.91) than in the experiential tasks (EQ Mean = -.79; HQ Mean =.08; Appeal Mean = -.69).

Table 3. Scale characteristics

\begin{tabular}{clrrrrr}
\hline \multirow{2}{*}{ Criterion } & \multicolumn{2}{c}{ Scale } & $\begin{array}{c}\text { Cronbach's } \\
\text { Alpha }\end{array}$ & \multicolumn{1}{c}{ Mean } & $\begin{array}{c}\text { Stand. } \\
\text { Dev. }\end{array}$ & \multicolumn{2}{c}{ Min. } & Max. \\
\hline \multirow{3}{*}{ Experiential Task } & EQ & .922 & -.79 & 1.17 & -2.63 & 1.63 \\
& HQ & .943 & .08 & 1.27 & -2.71 & 2.14 \\
& APPEAL & .958 & -.69 & 1.11 & -3.00 & 1.75 \\
\hline \multirow{3}{*}{ Goal-directed Task } & EQ & .840 & -1.362 & .89 & -3.00 & .63 \\
& HQ & .970 & -.507 & 1.41 & -3.00 & 2.43 \\
& APPEAL & .958 & -.91 & 1.11 & -2.88 & 1.75 \\
\hline
\end{tabular}

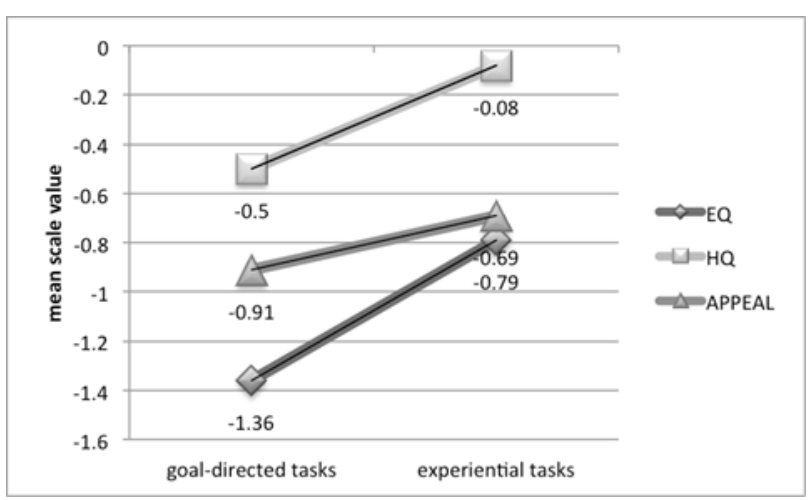

Figure 2. The ergonomic quality, hedonic quality, and appeal of the experiential tasks versus the goal-directed tasks. $\mathrm{EQ}=$ ergonomic quality, $\mathrm{HQ}=$ hedonic quality 
Figure 2 shows the differences for the EQ, HQ, and appeal between the experiential tasks and goal-directed tasks. According to the results, the HQ and EQ were experienced more in the goal-directed tasks than in the experiential tasks. The judgment of the appeal (APPEAL) is a comprehensive evaluation of the product and consists of the individual's perceptions of the EQ and HQ. A regression analysis was performed in order to see the experiential tasks' appeal from the experiential tasks' EQ and HQ, and the goal-directed tasks' appeal from the goal-directed tasks' EQ and HQ (see Table 4).

Table 4. Regression analysis of the ergonomic quality, hedonic quality, and ergonomic quality and hedonic quality interaction on appeal (for the experimental tasks and goal-directed tasks)

\begin{tabular}{|c|c|c|c|c|c|c|c|c|c|}
\hline & Criterion & Predictors & $\mathrm{R}^{2}$ & Adj. $R^{2}$ & Beta & Std. error & $\beta$ & t. & Sig. \\
\hline $\begin{array}{c}\text { Experiential } \\
\text { Task }\end{array}$ & Appeal & $\begin{array}{l}\text { EQ } \\
\text { HQ }\end{array}$ & .770 & .758 & $\begin{array}{l}.357 \\
.635\end{array}$ & $\begin{array}{l}.067 \\
.062\end{array}$ & $\begin{array}{l}.420 \\
.807\end{array}$ & $\begin{array}{r}5.303 \\
10.201\end{array}$ & $\begin{array}{l}.000 * * * \\
.000 * * *\end{array}$ \\
\hline $\begin{array}{c}\text { Goal- } \\
\text { directed } \\
\text { Task }\end{array}$ & Appeal & $\begin{array}{l}\text { EQ } \\
\text { HQ }\end{array}$ & .816 & .806 & $\begin{array}{l}.481 \\
.519\end{array}$ & $\begin{array}{l}.080 \\
.050\end{array}$ & $\begin{array}{l}.429 \\
.736\end{array}$ & $\begin{array}{r}6.024 \\
10.334\end{array}$ & $\begin{array}{l}.000 * * * * \\
.000^{* * * *}\end{array}$ \\
\hline
\end{tabular}

In the experiential tasks, a significant regression equation was found $(\mathrm{F}(2,37)=61.914, p<.001)$, with an $\mathrm{R}^{2}$ of .770. It was confirmed that EQ $(\mathrm{t}(40)=5.303, \mathrm{p}<.001)$ and HQ $(\mathrm{t}(40)=10.201, \mathrm{p}<.001)$ had an effect on appeal. HQ $(\beta=.807)$ contributed almost twice as much as the EQ $(\beta=.420)$ to the judgment of the appeal. The explanatory power explaining the dependent variable by the independent variables was $77 \%$. No interaction between the EQ and HQ was found. Similar results were found in goal-directed tasks. A significant regression equation was found $(\mathrm{F}(2,37)=82.166, p<.001)$ with an $\mathrm{R}^{2}$ of .816 in the goal-directed session. As a result of confirming the significance of each dimension, EQ $(\mathrm{t}(40)=6.024$, $\mathrm{p}<.001)$ and HQ $(\mathrm{t}(40)=10.334, \mathrm{p}<.001)$ affected on appeal. As with the experiential tasks results, the influence of HQ $(\beta=.736)$ on appeal was also higher than that of EQ $(\beta=.429)$. This result implies that the final judgment of the appeal depends more on the HQ than on the EQ for the TV-viewing experience in both situations. The implications of this will be described further in the DISCUSSION section. Similarly, the correlation analysis results showed a positive linear relationship between the judgement of appeal (APPEAL) and other UX quality dimensions (EQ and HQ) in the experiential session (APPEAL $x$ EQ: $r=.351^{*}$, APPEAL $x$ HQ: $\mathrm{r}=.771^{* *}$ ) and in the goal-directed session (APPEAL x EQ: $\mathrm{r}=.535^{* *}$, ATT x HQ: $\left.\mathrm{r}=.797^{* *}\right)$, respectively. In both sessions, the correlation between APPEAL and HQ was higher.

\section{CONCLUSION}

\subsection{Discussion}

In this paper, we investigated how the TV viewers experience differs in the experiential situation and the goal-directed situation. First, we confirmed that EQ and HQ are main factors of user experience measurements and independent each other, through factor analysis. Next, we measured whether the experience of both sessions were different each other. The results for the EQ, HQ, and appeal were significantly different according to whether participants were performing goal-directed tasks or experiential tasks; thus, H1 was supported. The hypothesis (H2) that users would be affected more in the goal-directed situation was also proved. As a result of the evaluation of the two sessions, TV viewers were more experienced in the goal-directed situation. Regression analysis showed that both EQ and HQ had a positive effect on APPEAL. The similar results were shown in the correlation analysis that a positive linear relationship between APPEAL and EQ and HQ in the experiential tasks and the goal-directed tasks. These results indicate again that the user perceive the product's character through ergonomic quality and the hedonic quality together. Interestingly, the results of the regression analysis showed that the contribution of the HQ was almost twice as much as the EQ for the APPEAL in both situations; thus, H3 was also supported. This implies the fundamental purpose of the user-TV interaction is to experience pleasure and achieving the 
goal is also the pleasure-seeking process as part of the fun. This also implies that hedonic aspect is the important factor than the ergonomic aspect on the TV-viewing experience regardless of the situation.

Based on the results of this study, the following constraints need to be discussed. As Chorianopoulos and Spinellis (2004) indicated, the interactive TV experience is influenced by both the interaction with the TV and the TV's contents. Although we tried to focus on the interaction with the TV itself, and minimized the impact of the TV's contents, it may be impossible to fully control the influence of the TV's contents in experiments. The two groups were exposed to different TV contents during the experiment. This implies that participants may have been influenced by the TV's contents while they were evaluating the experience in interacting with the TV. Another constraint is that the "interface" was controlled to focus on the "user experience." All tasks performed in this study were controlled with the four-way buttons of the remote control that have been used so far. We did not include personal computer-like features such as direct text input or pop-up windows. The main features of interactive TVs, so called "smart TV," are focused on its functions and its interface resembles a personal computer or smart phone user interface. This embarrasses users who are accustomed to traditional TV interfaces and they may feel pressured to learn a new way to use the interactive features.

\subsection{Conclusion}

In this study, we demonstrated that users had a different experience according to the different usage modes. Also, the TV viewers in this study reported having a "better experience" when they could achieve the goals. The goals mentioned here are mainly about pleasure rather than productivity. This finding means that the process of achieving the entertainment goals can also be a part of pleasure, and interactive TV can provide a better experience by providing various ways to experience pleasure corresponding to the user needs.

According to the results, TV viewers were more affected by the hedonic quality than by the ergonomic quality in both situations. TV viewers aspire to have fun and to relax; thus, the way of interacting with the TV should be more focused on enhancing the emotional qualities. As Vorderer (2001) mentioned, TV is not used for work but for entertainment, and research into the entertainment experience should examine ways of moderating the users' moods, focusing on their emotional outcomes.

It is necessary to determine how users' attitudes change based on their experience and the environment surrounding the TV, and to provide users with an optimized TV experience considering the changes in the TV. In particular, continuous and long-term observation is necessary, since hedonic aspects are known to increase as time goes on (Jordan 2000). Good emotions create a favorable experience, and the more that people experience, the deeper the relationship with the medium. In this sense, it is good that there is a growing number of studies employing long-term user experience measures (such as diaries, the experience sampling method, the day reconstruction method, and the user experience curve method) (Drouet and Bernhaupt, 2016; Kujala et al. 2011).

\section{REFERENCES}

Apter, M. 1989. Reversal theory: Motivation, emotion and personality. Florence, KY, US: Taylor \& Frances/Routledge.

Ardito, C. et al, 2008. Combining Quantitative and Qualitative Data for Measuring User Experience of an Educational Game. Proceedings of the International Workshop on Meaningful Measures: Valid Useful User Experience Measurement (VUUM): 27-31.

Bellman, S. et al, 2017. The effects of social TV on television advertising effectiveness. Journal of Marketing Communications, 23(1): 73-91.

Chorianopoulos, K. and Spinellis, D. 2004. User interface development for interactive television: Extending a commercial DTV platform to the virtual channel API. Computers and Graphics 28(2): 157-166.

Darnell, D. 2007. How do people really interact with TV?: naturalistic observations of digital tv and digital video recorder users. Computers in Entertainment (CIE) - Interactive TV 5(2) April/June 2007. doi:10.1145/1279540.1279550.

Drouet, D. and Bernhaupt, R., 2016. User experience evaluation methods: Lessons learned from an interactive TV case-study. In Human-Centered and Error-Resilient Systems Development (pp. 351-358). Springer, Cham. 
Dillon, A. 2002. Beyond Usability: Process, Outcome and Affect in human computer interactions. Canadian Journal of Library and Information Science 26(4):57-69.

Hartmann, J. et al, 2007. Investigating attractiveness in web user interfaces. In: Proceedings of the CHI 2007 Conference on Human Factors in Computing Systems. ACM, Addison-Wesley, New York. 387-396.

Hassenzahl, M. et al, 2000. Hedonic and Ergonomic Quality Aspects Determine a Software's Appeal. CHI '2000 Proceedings of the SIGCHI conference on Human Factors in Computing Systems. The Hague, Amsterdam. 201-208.

Hassenzahl, M. 2001. The Effect of Perceived Hedonic Quality on Product Appealingness. International Journal of Human-Computer Interaction, 13(4): 481-4.99.

Hassenzahl, M. et al, 2002. The importance of a software's pragmatic quality depends on usage modes. In: Luczak, H., Cakir, A.E., Cakir, G. (Eds.), Proceedings of the 6th international conference on Work with Display Units. WWDU, 2002: 275-276.

Hassenzahl, M. and Ullrich, D. 2007. To do or not do to: Differences in user experience and retrospective judgements depending on the presence or absence of instrumental goals. Interacting with Computers 19(4): 429-437.

Jordan, P. 2000. Designing pleasurable products: An Introduction to the New Human Factors. London: Taylor \& Francis.

Kujala, S. et al, 2011. UX Curve: A method for evaluating long-term user experience. Interacting with Computers 23(5): 473-483.

Lee, B. and Lee, R. 1995. How and why people watch TV: implications for the future of interactive television. Journal of Advertising Research (Nov-Dec 1995): 9.

Lekakos, G. et al, 2001. Information systems in the living room: A case study of personalized interactive TV design. Global Co-Operation in the New Millennium The 9th European Conference on Information Systems. Bled, Slovenia (June 27-29): 319-329.

Livaditi, J. et al, 2003. Needs and gratifications for interactive TV implications for designers. Proceedings of the 36th Annual Hawaii International Conference on System Sciences, 2003. DOI: 10.1109/HICSS.2003.1174237

Mano, H. and Oliver, R. 1993. Assessing the Dimensionality and Structure of the Consumption Experience: Evaluation, Feeling, and Satisfaction. Journal of Consumer Research 20(3): 451-466.

Morris, S. and Smith-Chaigneau, A. 2012. Interactive TV Standards: A Guide to MHP, OCAP, and JavaTV. CRC Press, Language Arts \& Disciplines.

Norman, D. 2004. Emotional Design: Why We Love (or Hate) Everyday Things. New York, Basic Books.

Novak, P. et al, 2003. The Influence of Goal-Directed and Experiential Activities on Online Flow Experiences. Journal of Consumer Psychology, 13 (1\&2): 3-16.

O'Sullivan, D. et al, 2004. Improving the Quality of the Personalized Electronic Program Guide. User Modeling and User-Adapted Interaction 14(1): 5-36.

Pirker, M.M. and Bernhaupt, R., 2011. June. Measuring user experience in the living room: results from an ethnographically oriented field study indicating major evaluation factors. In Proceedings of the 9th European Conference on Interactive TV and Video (pp. 79-82). ACM.

Sánchez-Franco M. and Roldán, J. 2005. Web acceptance and usage model: A comparison between goal-directed and experiential web users. Internet Research 15 (1): 21-48.

Tractinsky, N. et al, 2000. What is beautiful is usable. Interacting with Computers. 13(2): 127-145.

Tuch, A. et al, 2012. Is beautiful really usable? Toward understanding the relation between usability, aesthetics, and affect in HCI. Computers in Human Behavior 28, no. 5: 1596-1607.

Vorderer, P. 2001. It's all entertainment, sure. But what exactly is entertainment? Communication research, media psychology, and the explanation of entertainment experiences. Poetics 29, no. 4-5 (November 2001): 247-261. 